\title{
Earthly powers
}

In 1862, British physicist Lord Kelvin (William Thomson) tried to estimate the Earth's age, working from a simple theory of the cooling process from primordial times. He assumed a constant, temperatureindependent conductivity of the Earth's interior, and no internal sources of heat, arriving at an estimate of 98 million years. Thirty years later, another physicist, John Perry, pointed out that, should the Earth's thermal conductivity decrease with temperature, the estimated age might become much longer, even as long as billions of years. Perry was right.

The presence of radiogenic heating internal heat created by the decay of long-lived radioactive isotopes - was unknown to Kelvin, and had little to do with his poor estimate. Yet Kelvin was poking around some deep mysteries. Radiogenic heating does play an important role in the Earth's interior geophysical dynamics, and might even be a major driver of all mantle convection - responsible for volcanoes and plate tectonics. The evidence is only slowly accruing, as it relies on detecting the most elusive particles currently known - neutrinos.

Geophysicists estimate that the total heat flow outward from the Earth's interior to its surface is around 47 TW. Some of this heat is primordial, a remnant of the hot early conditions during the Earth's formation. The rest is clearly radiogenic, and yet the relative contribution of the two remains highly uncertain. We have no way to know how much primordial heat remains, and have only elusive neutrinos streaming from radioactive decays to tell us about the radiogenic contribution. Various models, based on assumed chemical and physical properties within the Earth's interior, yield estimates in the range of 15-41 TW for the radiogenic component and 12-30 TW for remaining primordial heat.

For the radiogenic component, the principal unknowns are the total rates of decay of ${ }^{232} \mathrm{Th}$ and ${ }^{238} \mathrm{U}$, which have been the focus of study by two international collaborations, KamLAND and Borexino. Both have been trying to detect antineutrinos from such decays geoneutrinos - and the KamLAND team made the first detection of such neutrinos ten years ago. Last month, the Borexino group published its latest findings (Phys. Rev. D 92, 031101(R); 2015), which point to higher radiogenic heating than

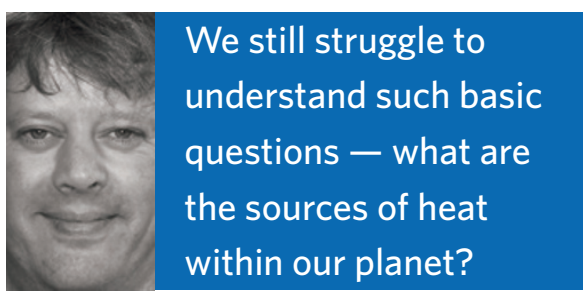

geoneutrinos come from within the Earth's mantle, rather than the crust. It seems to be about half.

Going one step further, the researchers also estimated the total amount of heat generated by radiogenic heating, finding about 33 TW (with large error bars) - higher than earlier studies. This might actually be enough for radiogenic heating alone - even in the absence of significant primordial heat - to drive mantle convection.

However, these results aren't wholly independent of theory. They require an estimate of the number of neutrinos coming from the Earth's crust, and this is based on the measured abundance of uranium and thorium in crustal samples. Subtracting this signal leaves the mantle contribution. It also requires a model-dependent inference from the detected geoneutrino flux to the distribution of radioactive isotopes throughout the earth. One interesting aspect of this ongoing work - suggested to me by Jason Detwiler of the KamLAND group is that there is a small disagreement persisting between the Borexino and KamLAND results for the number of mantle geoneutrinos.

So far, the KamLAND results are consistent with a slightly lower contribution from the mantle than the Borexino results. The disagreement has persisted as each group has updated their findings (KamLAND in 2013 and Borexino now). What will happen next? It might very well be that the distinct geology of the two sites in Japan and Italy - may account for these differences, and they'll disappear as more becomes known about those differences. Or, more interestingly, the disagreement could grow, pointing to fundamental problems or errors in the models used to interpret the geoneutrino results.

Next up will come the SNO+ collaboration, operating a detector located some $2 \mathrm{~km}$ underground in Sudbury, Canada. This should help get even more accurate estimates of the true rate of radioactivity within the Earth. It's surprising in one sense that we still struggle to understand such basic questions - what are the sources of heat within our planet? Yet finding answers turns out to demand the most sensitive detectors we can muster, using the most advanced physics.

\section{MARK BUCHANAN}

